\title{
Effects of topical treatment of foot rot in sheep using ozonated olive ointment
}

\author{
Tomasz Szponder ${ }^{1}$, Joanna Zdziennicka ${ }^{2}$, Aneta Nowakiewicz ${ }^{3}$, Michał Świeca ${ }^{4}$, \\ Aleksandra Sobczyńska-Rak ${ }^{1}$, Beata Żylińska ${ }^{1}, \mathrm{Krzysztof} \mathrm{Patkowski}^{5}$, \\ Andrzej Junkuszew ${ }^{5}$, Joanna Wessely-Szponder ${ }^{2 凶}$
${ }^{1}$ Department and Clinic of Animal Surgery, Faculty of Veterinary Medicine, University of Life Sciences, 20-612 Lublin, Poland ${ }^{2}$ Sub-Department of Pathophysiology, Department of Preclinical Veterinary Sciences,
${ }^{3}$ Sub-Department of Veterinary Microbiology, Department of Preclinical Veterinary Sciences, Faculty of Veterinary Medicine, University of Life Sciences, 20-033 Lublin, Poland
${ }^{4}$ Department of Biochemistry and Food Chemistry, University of Life Sciences, 20-704 Lublin, Poland
${ }^{5}$ Faculty of Animal Sciences and Bioeconomy, Institute of Animal Breeding and Biodiversity Conservation, University of Life Sciences, 20-950 Lublin, Poland \\ joanna.wessely@up.lublin.pl
}

Received: March 24, $2021 \quad$ Accepted: August 27, 2021

\begin{abstract}
Introduction: Foot rot in small ruminants is highly contagious, causes severe lameness, and impairs fertility and wool and meat production. It is usually treated with parenteral antibiotics, with attendant antibiotic resistance risk, and with bactericidal footbaths, potentially harmful to humans and the environment. An alternative treatment in sheep is proposed based on repeated topical ozonated ointment application. Its effectiveness and safety were evaluated by estimation of acute-phase response, biochemical indicators of organic damage, and antioxidant/oxidant balance (AOB). Material and Methods: The study was conducted on ten sheep with Egerton scale 2-3 lesions. Ozone application was repeated every day for seven days. Blood was drawn first (T0) after foot cleaning and before ozonation, then (T1) seven days after the first ozone application, and finally (T2) four days after the last application. Results: High clinical effectiveness was observed, with total recovery by 28 days from the start of treatment. A significant increase in antiradical activity was noted on the basis of a 2,2'-azino-bis(3-ethylbenzothiazoline-6sulphonic acid) assay from $1.16 \pm 0.04 \mu \mathrm{molTe} / \mathrm{mL}$ at $\mathrm{T} 0$ to $1.23 \pm 0.03 \mu \mathrm{molTe} / \mathrm{mL}$ at $\mathrm{T} 1$, with a slight decrease in oxidative stress. Calculated on the basis of antiradical capacity, AOB was higher at $\mathrm{T} 1(130 \pm 19 \%)$ and decreased to $110 \pm 16 \%$ at $\mathrm{T} 2$. Calculated on the basis of reducing power, it was $169 \pm 22 \%$ at $\mathrm{T} 1$ and $131 \pm 17 \%$ at $\mathrm{T} 2$. Conclusion: These results indicated that the AOB is efficient enough to prevent oxidative organ injury and the applied doses of ozone are safe for animals.
\end{abstract}

Keywords: foot rot, sheep, ozonated olive ointment, antioxidant/oxidant balance.

\section{Introduction}

Virulent foot rot is a highly contagious, widely distributed disease that affects productivity and impairs small ruminants' welfare worldwide. This condition involves the interaction between the obligate anaerobic bacteria Dichelobacter nodosus and Fusobacterium necrophorum causing severe lameness combined with reduced wool and meat production and decreased fertility $(1,3)$. Treatment programs usually include the parenteral administration of antibiotics such as oxytetracycline and enrofloxacin in combination with footbaths in bactericidal solutions and antibiotic aerosol spray (12). However, this treatment has some disadvantages. Overuse and misuse of antibiotics leads to the development of drug resistance (23), and chemical solutions used for bathing feet are toxic to both the human personnel and the environment. Additionally, the stringent limitation on the use of antibiotics in agri-environment schemes and on organic farms forces the introduction of alternative treatment schemes for foot $\operatorname{rot}(14)$.

The concept of using ozone to improve the healing of infected wounds, and necrotic or poorly oxygenated tissue has been widely explored in orthopaedics, dental medicine and with skin wounds $(6,18)$. Ozone $\left(\mathrm{O}_{3}\right)$ is the trivalent form of oxygen with high reactivity and solubility in water and plasma; moreover, some authors 
indicated its antioxidant potency (5). Ozone appeared to be a highly reactive molecule which exhibited strong bactericidal, fungicidal, antivirus, anti-yeast and antiprotozoa activities (8). To date, ozone therapy has been successfully used in infected wounds, chronic skin ulcers, early-stage gangrene and advanced ischaemic diseases (11). There are some products which are used in veterinary medicine, such as ozonated vegetable oil with lenitive, emollient, cicatrising, antibacterial and hygiene-maintaining action in the form of creams, gases, syringes, foams, and boluses (8). Previous successful applications of ozone in veterinary medicine were described in sheep in treatment of foot rot (25) and in other animal species in many disorders, such as mastitis, metritis, endometritis, foetal membrane retention, vaginitis, urovagina, enteritis and laminitis (8). In our previous work, ozonated saline was applied for local treatment of ovine foot rot and this proved to be safe and highly effective (25). However, the preparation of the solution ex tempore was time-consuming; therefore, to eliminate this disadvantage an ozonated olive oil ointment (Ozonsept) was introduced to the treatment regime. To our knowledge, to date there are no published works about the use of this preparation in the treatment of foot rot in sheep.

The purpose of this study was to evaluate the ozonated olive oil ointment as an easily applied therapeutic with a stable ozone concentration. The effectiveness of this therapeutic option in treatment of sheep was assessed on the clinical outcome, whereas the safety was investigated on the basis of oxidative stress response, antioxidant defence, markers of potential organic damage and systemic inflammatory reaction.

\section{Material and Methods}

The study was conducted on ten adult female Polish lowland sheep of the Uhruska variety weighing approximately $40-50 \mathrm{~kg}$, aged between 1 and 2 years and maintained on the Bezek experimental farm of the University of Life Sciences in Lublin. The control group consisted of five healthy female sheep of the same breed and similar age and weight. All the animals were fed, housed and cared for in accordance with Directive 2010/63/EU on animal care.

The clinical parameters of rectal temperature, pulse and respiratory rate were recorded at the times of blood sampling. Acute foot rot diagnosis was based on the typical clinical signs such as lameness, lesions with interdigital inflammation, hoof horn separation, a characteristic foul smell, and absence of hyperplasia of the sole, according to Raadsma and Egerton (20).

After assignation to the experimental group $(\mathrm{n}=10)$, each sheep was treated and underwent cleaning and gentle removal of the necrotic tissue as the first step. As further treatment, an ozonated olive oil ointment (Ozonosept, Metrum Cryoflex, Łomianki, Poland) containing ozone at $100 \mathrm{mg} / \mathrm{g}$ was used. The ointment was applied using a small brush. Then, each sheep was left on a clean solid surface for $30 \mathrm{~min}$ for the preparation to be absorbed. The procedure of ozone application was repeated once a day every day for seven consecutive days. Careful clinical examination was performed before each ozone administration, at the T2 time point (day 11), on the 14th day after beginning therapy, and when it was finished (Fig. 1).

Material was taken from each animal as lesion swabs and collected in Amies transport medium (Argenta, Poznań, Poland). Samples were delivered to the laboratory of the Sub-Department of Veterinary Microbiology in the Faculty of Veterinary Medicine of the University of Life Sciences on ice and DNA was immediately extracted. DNA was isolated with a Tissue \& Bacterial DNA Purification Kit (EURx, Gdańsk, Poland) according to the manufacturer's instructions, and direct PCR testing was conducted according to conditions described by McPherson et al. (16).

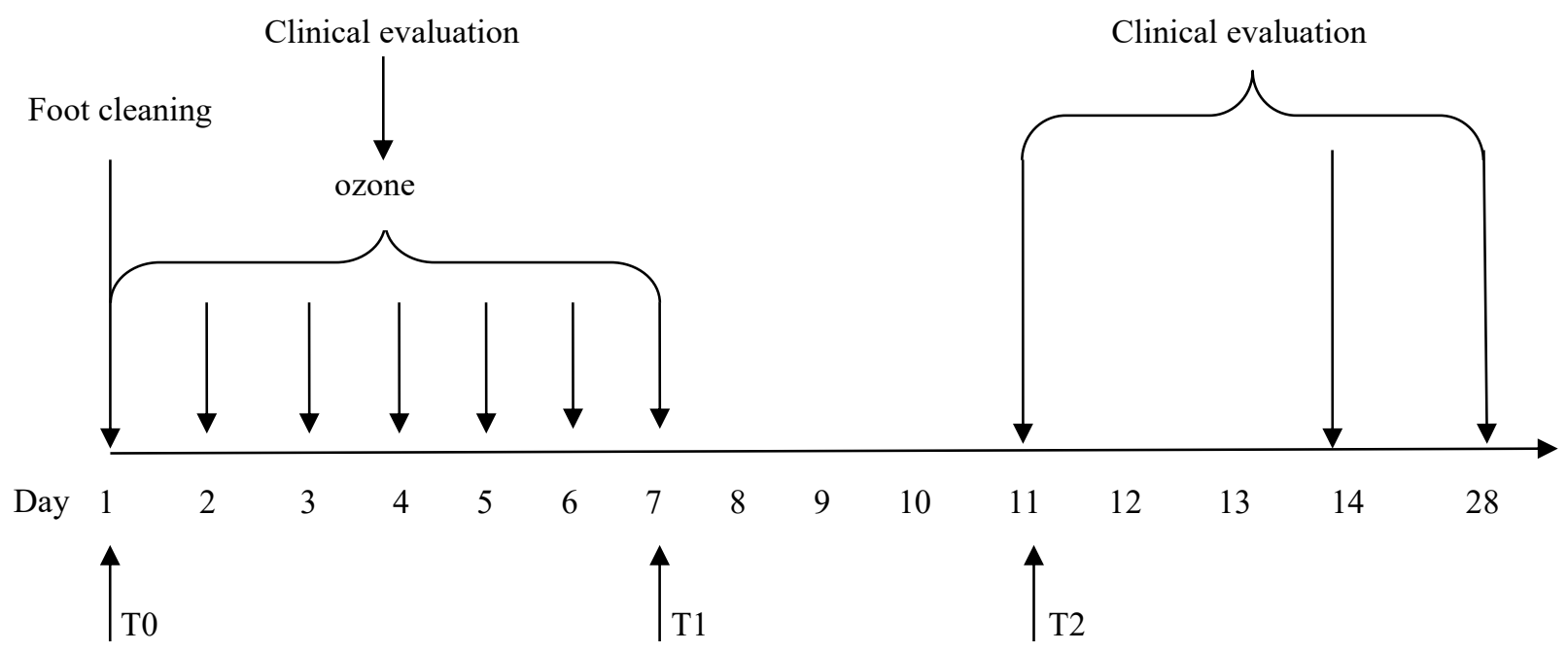

T0 - first blood sampling; T1 - second blood sampling; T2 - third blood sampling

Fig. 1. Timeline for clinical evaluation and blood sampling 
The PCR reaction was carried out using $2 \mu \mathrm{L}$ of extracted material, $5 \mu \mathrm{L}$ of Gold Taq Mix (Syngen Biotech, Wrocław, Poland), and 50 pmol of each primer, which was designed by Genomed (Warsaw, Poland), in a T100 thermal cycler (Bio-Rad, Hercules, CA, USA). Bacterial DNA isolated from D. nodosus ATCC25549 was used as the positive control.

Blood was drawn at the following time points: first at T0, immediately after foot cleaning and before ozone treatment, then at $\mathrm{T} 1$, seven days after the first ozone application, and finally at T2, four days after the last application of ozone (day 11). Complete blood cell counts were performed using the Abacus Junior Vet analyser (Diatron, Budapest, Hungary) at the same time points (T0, T1 and T2) at which blood samples were obtained for other analyses.

The plasma concentration of fibrinogen (in $\mathrm{g} / \mathrm{L}$ ) was assessed with the heat precipitation method, and the haptoglobin plasma concentration was estimated on the basis of methaemoglobin reduction (25). As biochemical indicators of organic damage, alanine aminotransferase (ALT), aspartate aminotransferase (AST), total bilirubin (BIL T) urea and creatinine (CREA) were analysed using an automatic apparatus as described by Guanche et al. (10) at the T0 and T2 time points and compared to those of healthy sheep.

The free radical scavenging ability was measured using the improved 2,2'-azino-bis(3-ethylbenzothiazoline-6-sulphonic acid) (ABTS) decolourisation assay and expressed as Trolox equivalents (Te) in $\mathrm{mg}$ per $\mathrm{mL}$ using the protocol of Re et al. (21). The reducing power (RP) was determined following Oyaizu's method (17) and expressed as Trolox equivalents (Te) in mg per $\mathrm{mL}$. The plasma concentration of malondialdehyde (MDA) was measured using the spectrophotometric method on the basis of the reaction between MDA and thiobarbituric acid. Concentrations of MDA were estimated on the basis of the standard curve obtained using malondialdehyde bis (dimethyl acetal) and expressed as nmol/mL (25). To compare antioxidant/oxidant balance (AOB) values of plasma before and after ozone treatment, the antioxidant capacity (AC)/peroxide level ratio was calculated as the ABTS/MDA and RP/MDA ratios. Data were expressed as $(\%)$ of the T0 value. The calculation was carried out individually for each sheep enrolled in the study at both time points (13).

Statistical analysis. Data were presented as mean \pm standard deviation for continuous variables. Significance was identified by one-way ANOVA using Statistica software version 13.1 (Dell Technologies, Round Rock, TX, USA). Differences were considered statistically significant at $\mathrm{P}<0.05$.

\section{Results}

Ovine foot rot was confirmed on the basis of clinical evaluation and the molecular test. The measured haematological and clinical parameters, i.e. rectal temperature, pulse and respiratory rate were within the physiological ranges (data not shown). Dichelobacter nodosus was confirmed in all samples tested by the presence of an amplification product of the variable region of 16s rRNA gene with appropriate molecular weight (783bp) visualised under UV light after staining with SimplySafe (EURx, Gdańsk, Poland) in a $2 \%$ agarose gel (Fig. 2).

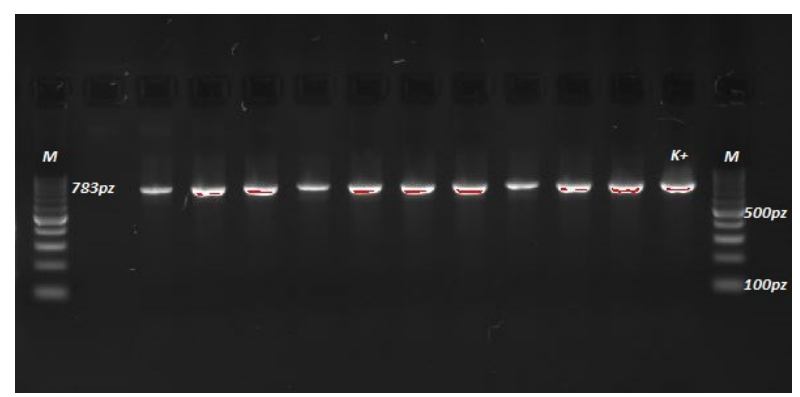

Fig 2. Amplification product of the variable region of $16 \mathrm{~s}$ rRNA (783pz) of Dichelobacter nodosus from samples tested

*K+ Dichelobacter nodosus ATCC25549

Table 1. Changes in plasma concentration of selected acute-phase proteins in the course of local ozone treatment in sheep $(\mathrm{n}=10)$

\begin{tabular}{lcccc}
\hline Protein & Control & Day 0 & Day 7 & Day 11 \\
\hline Fibrinogen $(\mathrm{g} / \mathrm{L})$ & $4.10 \pm 1.00$ & $4.97 \pm 0.51$ & $4.67 \pm 0.61^{*}$ & $5.06 \pm 0.61^{*}$ \\
Haptoglobin $(\mathrm{g} / \mathrm{L})$ & $0.22 \pm 0.20$ & $0.61 \pm 0.13$ & $0.35 \pm 0.11^{*}$ & $0.67 \pm 0.16^{*}$ \\
\hline
\end{tabular}

$* \mathrm{P}<0.05$ in comparison with value on day 0

Table 2. Antioxidant capacity, antioxidant/ oxidant balance, oxidative status

\begin{tabular}{|c|c|c|c|c|c|}
\hline Measurement & ABTS $(\mu \mathrm{molTe} / \mathrm{mL})$ & $\mathrm{RP}(\mu \mathrm{molTe} / \mathrm{mL})$ & MDA (nmol/mL) & AOB by ABTS ( $\%$ of T0) & AOB by RP $(\%$ of $T 0)$ \\
\hline Control & $1.22 \pm 0.01$ & $0.14 \pm 0.02$ & $6.2 \pm 0.18$ & - & - \\
\hline T0 & $1.16 \pm 0.04$ & $0.1 \pm 0.02$ & $7.84 \pm 0.18$ & - & - \\
\hline $\mathrm{T} 1$ & $1.23 \pm 0.03^{*}$ & $0.14 \pm 0.01 *$ & $6.45 \pm 0.39$ & $129.93 \pm 19.1$ & $169.2 \pm 22.0$ \\
\hline $\mathrm{T} 2$ & $1.2 \pm 0.07$ & $0.13 \pm 0.01 *$ & $7.4 \pm 0.2$ & $110.00 \pm 16.2$ & $130.77 \pm 17.0$ \\
\hline
\end{tabular}

ABTS - 2,2'-azino-bis(3-ethylbenzothiazoline-6-sulphonic acid); RP - reducing power; MDA - malondialdehyde; AOB - antioxidant/oxidant balance; $* \mathrm{P}<0.05$ in comparison with $\mathrm{T} 0$ 
All lesions healed completely within 28 days and all sheep recovered fully from lameness and the without suffering any adverse effects of the ointment during treatment. Immediately after ozone treatment, at the T1 measurement, plasma concentrations of fibrinogen and haptoglobin significantly decreased, but subsequently at the T2 measurement they increased; however, all values were within the normal range (Table 1), as biochemical assays revealed. The differences between concentrations at successive time points were insignificant (data not shown).

On the basis of the ABTS assay, we estimated a significant $(\mathrm{P}<0.01)$ increase in antiradical activity seven days after the start of ozone treatment. The obtained values elevated from $1.16 \pm 0.04 \mu \mathrm{molTe} / \mathrm{mL}$ at the first measurement (T0) to $1.23 \pm 0.03 \mu \mathrm{molTe} / \mathrm{mL}$ at the second (T1). The changes at the third measurement (T2) were insignificant in comparison with those at T0 and $\mathrm{T} 1$. The reducing power was significantly higher at the second (T1) and third (T2) measurements $(\mathrm{P}<0.05)$ than it was at the initial one (T0). The plasma concentration of MDA slightly decreased at the T1 and T2 measurements in comparison with T0, but without statistical significance. When calculated on the basis of antiradical capacity (AC), AOB was higher at T1 $(130 \pm 19 \%)$ and decreased to $110 \pm 16 \%$ at $\mathrm{T} 2$. When calculated on the basis of RP, it was $169 \pm 22 \%$ at T1 and $131 \pm 17 \%$ at $\mathrm{T} 2$ (Table 2).

\section{Discussion}

In the present study, confirmation of infection of sheep with Dichelobacter nodosus was obtained using a PCR test because of the reliability of this method's results. Foot rot results from the invasion of the epidermal tissue of the hooves by a complex mixture of bacteria, in which $D$. nodosus is considered a determining component. Some authors recommended PCR-based methods because confirmation via microbiological culture of $D$. nodosus is time-consuming and the culturing process requires practical qualifications and experience, which may extend the time needed to make a diagnosis and decision about the appropriate treatment; culturing can be particularly difficult when the sample site is contaminated with other pathogens. For these reasons, PCR seems to be the better option. Moreover, the method used allows both detection of genetic material in samples taken directly and confirmation of species affiliation in in vitro culture (16).

Previous appraisal of treatment with ozone found it to be a versatile bio-oxidative therapy in which ozone is possible to administer in different ways. The therapeutic activity of ozonated oil was mostly derived from its antibacterial, antihypoxic, analgaesic and immunomodulatory effects (6). The ozonated oil ointment used in our study proved to be an effective and convenient means for topical treatment of foot rot in sheep enabling precise administration and causing no undesirable effects.
Application of ozonated oil ointment in the present study brought about total recovery within 28 days without use of antibiotics in all eight sheep, all with an initial score between 2 and 3 on the Egerton scale (20). Moreover, the time of recovery was similar to those described by Ansari et al. (1) after usage of different antimicrobial therapies.

In our previous study, we introduced an alternative treatment scheme consisting of ozone therapy and the application of autologous platelet-rich plasma (PRP) as a source of high concentrations of growth factors and other bioactive molecules to enhance healing (25). However, that scheme is more complicated and needs preparation of autologous PRP, which is expensive and time-consuming and limits its large-scale use.

Ozonated saline concentrated to $70 \mathrm{mg} / \mathrm{mL}$ was applied in the authors' previous experiment (25), whereas in the current study oil ointment with ozone concentration of $100 \mathrm{mg} / \mathrm{mL}$ was used. Some other authors stressed that because repeated high concentration ozone applications may cause excessive oxidative stress, leading to changes in membrane permeability, enzyme inactivation and potential organ injury, they should be carefully evaluated before being introduced into clinical use (10). The blood toxicity indices ALT, AST, BIL and UREA can be recognised as markers of organic damage. According to Guanche et al. (10) in their study on rabbits treated with an ozone/oxygen mixture, these indices did not rise significantly. Similarly, in our experiment on sheep these biomarkers were likewise not significantly changed during treatment. These results provide evidence that ozonated ointment did not damage organs or alter the metabolism of the organism at the dose used.

In our experiment, we did not observe any side effects or significant changes in haematological parameters after treatment. These parameters after ozone application were previously evaluated by Bocci (2), who reported no modification of the erythrocytic volume due to osmotic swelling or haemolysis, and by Carvalho et al. (4), who noted only a mild response in WBC in the course of foot rot in crossbred Santa Inês sheep.

In the course of the experiment we also assessed the concentrations in plasma of two acute-phase proteins (APP), namely fibrinogen and haptoglobin, as indicative APPs in sheep (19), and we observed a significant decrease in both these markers after ozone treatment. A similar effect was noted by Fernandez-Cuadros et al. (9) after ozone treatment of osteoarthritis in humans. According to Carvalho et al. (4), the fibrinogen, haptoglobin and $\alpha 1$ acid glycoprotein APP concentrations did not increase in the course of foot rot in sheep. Another author stated that 24, 48 and 96 hours after therapy with ozone, the serum APP level remained constant (2). Additionally, according to Curro et al. (6), ozonated olive oil is non-toxic, accelerates wound healing and exerts anti-inflammatory effects.

We found a significant $(\mathrm{P}<0.01)$ increase in plasma antioxidant activity seven days after the start of ozone treatment on the basis of the ABTS assay and 
reducing power. The obtained values rose from $1.16 \pm 0.04 \mu \mathrm{molTe} / \mathrm{mL}$ at the first measurement (T0) to $1.23 \pm 0.03 \mu \mathrm{molTe} / \mathrm{mL}$ at the second measurement (T1). It was stated previously that in environments characterised by a protonic increase, such as ischaemic, hypoxic, or damaged tissues, ozonated olive oil releases molecular oxygen, driving the production of reactive oxygen species and generation of moderate oxidative stress (6). This oxidative stress activates antioxidant defence systems to eliminate these toxic compounds (11), and this activated condition promotes release of growth factors, triggers local antioxidant mechanisms, and repairs tissue (6). The potent antioxidant capacity of blood exposed to a small and precisely calculated dose of ozone can modulate the endogenous antioxidant system and aid in the control of different pathological conditions (5).

In our experiment, plasma concentration of MDA slightly and insignificantly decreased at the T1 and T2 measurements in comparison with the $\mathrm{T} 0$ concentration. Ozone generally generates the formation of radicals and promotes lipid peroxidation, but when administered in a controlled way in minor doses its promotion of peroxidation can be limited in order that it only activates certain routes to modify the immunological response (10). Thus, according to Martínez-Sánchez et al. (15), the paradoxical antioxidant effect of ozone therapy decreases the generation of MDA, the marker of lipid peroxidation. For more accurate evaluation of the balance between oxidant and antioxidant activity, the AOB was calculated. Consideration of this balance is a novel approach described by Laus et al. (13) for precise evaluation of serum antioxidant status, and this method demonstrated high versatility and applicability both in human and animal studies (24). In our experiment, AOB calculated on the basis of AC indicated a higher antioxidant value at $\mathrm{T} 1(129.93 \pm 19.10 \%)$, which decreased to $110 \pm 16.20 \%$ at T2. Similar increases of AOB calculated on the basis of RP were observed at T1 $(169.2 \pm 22.0 \%)$ in comparison with T0 $(100 \%)$ and to a lesser degree at T2 $(130.77 \pm 17.0 \%)$. The simultaneous evaluation of $\mathrm{AC}$ (on the basis of ABTS or RP) and MDA concentration (as "peroxide level") allows the estimation of oxidative balance in a manner impossible when considering AC alone. In a study by Reddy et al. (22) in haemodialysis patients, both plasma MDA level and RP decreased during the oxidative stress generated by the treatment. In our previous experiment (25), after the ozone therapy, AOB determined on the basis of the ABTS increased at the second measurement, whereas AOB determined on the basis of RP decreased. In the current experiment, due to the use of a different method of ozone application, generation of oxidative stress was lower and the obtained results indicated higher antioxidant capacity.

This research is a continuation and extension of our previous study and concerns the application of ozonated olive oil in the treatment of ovine foot rot. Our research confirmed the high effectiveness of the preparation.
Moreover, we did not observe any adverse effects, excessive oxidative stress or organ damage. In the course of treatment, the antioxidant system established was productive of a sufficient post-ozone therapy antioxidant/oxidant balance to prevent systemic inflammatory response and excessive oxidative stress that might have caused organ injury. Thus, the applied formulation and doses of ozone in oil ointment appear to be safe for animals. This approach seems to be a promising option for the treatment of foot rot in sheep, since the ointment is characterised by good therapeutic results, simple application and low cost.

Conflict of Interests Statement: The authors declare that there is no conflict of interests regarding the publication of this article.

Financial Disclosure Statement: This research was financed by the Faculty of Veterinary Medicine, University of Life Sciences in Lublin.

Animal Rights Statement: None required.

\section{References}

1. Ansari M.M., Dar K.H., Tantray H.A., Bhat M.M., Dar S.H., Naikoo M.: Efficacy of different regimens for acute foot rot in adult sheep. J Adv Vet Anim Res 2014, 1, 114-118, doi: 10.5455/javar.2014.a16.

2. Bocci V.: Oxygen-Ozone Therapy: A Critical Evaluation. Springer Science+Business Media, Dordrecht 2002.

3. Burke J.M., Parker C.F.: Effect of breed on response to foot rot treatment in mature sheep and lambs. Small Rumin Res 2007, 71, 165-169, doi: 10.1016/j.smallrumres.2006.06.003.

4. Carvalho V.S., Araújo B.R., Vasconcelos T.C., Chiminazzo C., Costa Neto A.O., Ayres M.C.C., Guimaräes J.E., Costa J.N.: Clinical evolution and evaluation of leukocyte parameters and acute phase protein of ovine footrot (in Portuguese). Pesq Vet Brasil 2012, 32, 1289-1296, doi: 10.1590/S0100736X2012001200013.

5. Clavo B., Rodríguez-Esparragón F., Rodríguez-Abreu D., Martínez-Sánchez G., Llontop P., Aguiar-Bujanda D., Fernández-Pérez L., Santana-Rodríguez N.: Modulation of Oxidative Stress by Ozone Therapy in the Prevention and Treatment of Chemotherapy-Induced Toxicity: Review and Prospects. Antioxidants 2019, 8, 588, doi: 10.3390/antiox8120588.

6. Currò M., Russo T., Ferlazzo N., Caccamo D., Antonuccio P., Arena S., Parisi S., Perrone P., Ientile R., Romeo C., Impellizzeri P.: Anti-inflammatory and Tissue Regenerative Effects of Topical Treatment with Ozonated Olive Oil/ Vitamin E Acetate in Balanitis Xerotica Obliterans. Molecules 2018, 23, 645, doi: 10.3390/molecules23030645.

7. Du Plessis L.H., van der Westhuizen F.H., Kotze H.F.: The protective effect of plasma antioxidants during ozone autohemotherapy. Afr J Biotechnol 2008, 7, 2472-2477, doi: 10.5897/AJB07.672

8. Duričić D., Valpotić H., Samardžija M.: Prophylaxis and therapeutic potential of ozone in buiatrics: Current knowledge. Anim Reprod Sci 2015, 159, 1-7, doi: 10.1016/j.anireprosci. 2015.05.017.

9. Fernandez-Cuadros M.E., Perez-Moro O.S., Albaladejo-Florin M.J., Algarra-Lopez R.: Ozone decreases biomarkers of inflammation (C-reactive protein and erythrocyte sedimentation rate) and improves pain, function and quality of life in knee osteoarthritis patients: A before-and-after study and review of the literature. 
Middle East J Rehabil Health Stud 2008, 5, e64507, doi: 10.5812/mejrh.64507.

10. Guanche D., Zamora Z., Hernández F., Mena K., Alonso Y., Roda M., Gonzáles M., Gonzales R.: Effect of ozone/oxygen mixture on systemic oxidative stress and organic damage. Toxicol Mech Methods 2009, 20, 25-30, doi: 10.3109/15376510903503107.

11. Güven A., Gundoğdu G., Vurucu S., Uysal B., Oztas E., Ozturk H., Korkmaz A.: Medical ozone therapy reduces oxidative stress and intestinal damage in an experimental model of necrotizing enterocolitis in neonatal rats. J Pediatr Surg 2009, 44, 1730-1735, doi: 10.1016/j.jpedsurg.2009.01.007.

12. Kaler J., Wani S.A., Hussain I., Beg S.A., Makhdoomi M., Kabli Z.A., Green L.E.: A clinical trial comparing parenteral oxytetracycline and enrofloxacin on time to recovery in sheep lame with acute or chronic footrot in Kashmir, India. BMC Vet Res 2012, 8, 12, doi: 10.1186/1746-6148-8-12.

13. Laus N.M., Soccio M., Alfarano M., Pasqualone A.S., Lenucci M.S., Di Miceli G., Pastore D.: Different effectiveness of two pastas supplemented with either lipophilic or hydrophilic/phenolic antioxidants in affecting serum as evaluated by the novel Antioxidant/Oxidant Balance approach. Food Chem 2017, 221, 278-288, doi: 10.1016/j.foodchem.2016.10.050.

14. Manyi-Loh Ch., Mamphweli S., Meyer E., Okoh A.: Antibiotic Use in Agriculture and Its Consequential Resistance in Environmental Sources: Potential Public Health Implications. Molecules 2018, 23, 795, doi: 10.3390/molecules23040795.

15. Martínez-Sánchez G., Delgado-Roche L., Díaz-Batista A., Pérez-Davison G., Re L.: Effects of ozone therapy on haemostatic and oxidative stress index in coronary artery disease. Eur J Pharmacol 2012, 691, 156-162, doi: 10.1016/j.ejphar.2012.07.010.

16. McPherson A. S., Dhungyel O.P., Whittington R.J.: Detection and Serogrouping of Dichelobacter nodosus Infection by Use of Direct PCR from Lesion Swabs To Support Outbreak-Specific Vaccination for Virulent Footrot in Sheep. J Clin Microbiol 2018, 56, e01730-17, doi: 10.1128/JCM.01730-17.
17. Oyaizu M.: Studies on products of browning reaction Antioxidative activities of products of browning reaction prepared from glucosamine. Japanese J Nutr 1986, 44, 307-315, doi: 10.5264/eiyogakuzashi.44.307.

18. Ozbay I., Ital I., Kucur C., Akcılar R., Deger A., Aktas S., Oghan F.: Effects of ozone therapy on facial nerve regeneration. Braz J Otorhinolaryngol 2017, 83, 168-175, doi: 10.1016/j.bjorl.2016.02.009.

19. Piccione G., Casella S., Giannetto C., Giudice E., Fazio F.: Utility of acute phase proteins as biomarkers of transport stress in ewes. Small Rumin Res 2012, 107, 167-171, doi: 10.1016/j.smallrumres.2012.05.008.

20. Raadsma H.W., Egerton J.R.: A review of footrot in sheep: Aetiology, risk factors and control methods. Livestock Sci 2013, 156, 106-114, doi: 10.1016/j.livsci.2013.06.009.

21. Re R., Pellegrini N., Proteggente A., Pannala A., Yang M., Rice-Evans C.: Antioxidant activity applying an improved ABTS radical cation decolorization assay. Free Radic Biol Med 1999, 26, 1231-1237, doi: 10.1016/S0891-5849(98)00315-3.

22. Reddy P.E., Manohar S.M., Reddy S.V., Bitla A.R., Vishnubhotla S., Rao S.P.V. Lakshmi Narasimha: Ferric reducing ability of plasma and lipid peroxidation in hemodialysis patients: intradialytic changes. Int J Nephrol Urol 2010, 2, 414-421.

23. Sierra J.M., Fusté E., Rabanal F., Vinuesa T., Viñas M.: An overview of antimicrobial peptides and the latest advances in their development. Expert Opin Biol Ther 2017, 17, 663-676, doi: 10.1080/14712598.2017.1315402.

24. Soccio M., Laus M.N., Flagella Z., Pastore D.: Assessment of Antioxidant Capacity and Putative Healthy Effects of Natural Plant Products Using Soybean Lipoxygenase-Based Methods. An Overview. Molecules 2018, 23, 3244, doi: 10.3390/molecules23123244.

25. Szponder T., Wessely-Szponder J., Świeca M., Smolira A., Gruszecki T.: The combined use of ozone therapy and autologous platelet-rich plasma as an alternative approach to foot rot treatment in sheep. A preliminary study. Small Rumin Res 2017, 156, 50-56, doi: 10.1016/j.smallrumres.2017.08.015. 